ENCYCIOPEDDE Encyclopédie berbère

BERBERE

28-29 | 2008

28-29 | Kirtēsii - Lutte

\title{
Leucochroa Candidissima Draparnaud
}

C. Roubet et S. Hachi

\section{OpenEdition}

Journals

Édition électronique

URL : http://journals.openedition.org/encyclopedieberbere/328

DOI : 10.4000/encyclopedieberbere.328

ISSN : 2262-7197

\section{Éditeur}

Peeters Publishers

\section{Édition imprimée}

Date de publication : 1 janvier 2008

Pagination : 4366-4374

ISBN : 2-7449-0707-4

ISSN : 1015-7344

\section{Référence électronique}

C. Roubet et S. Hachi, «Leucochroa Candidissima Draparnaud », Encyclopédie berbère [En ligne], 28-29 | 2008, document L16, mis en ligne le 01 juin 2013, consulté le 25 septembre 2020. URL : http:// journals.openedition.org/encyclopedieberbere/328 ; DOI : https://doi.org/10.4000/ encyclopedieberbere.328

Ce document a été généré automatiquement le 25 septembre 2020.

(c) Tous droits réservés 


\title{
Leucochroa Candidissima Draparnaud
}

\author{
C. Roubet et S. Hachi
}

\section{Sa présence et son rôle en milieu anthropique préhistorique}

1 Ce mollusque gastéropode terrestre très commun sur le pourtour méditerranéen fut récolté par les dernières populations préhistoriques du Maghreb. Il a fait partie de leur alimentation saisonnière durant plus de quinze mille ans et pourrait contribuer à préciser les données paléo-environnementales et comportementales mises en place avant et pendant l'Holocène, en s'appuyant sur d'autres espèces terrestres.

2 L'espèce actuelle, comparable à la forme fossile, semble n'avoir pas changé. Son écologie et sa biogéographie sont dépendantes des formations végétales de terrains calcaires, karstiques, telliens et atlasiques (jusqu'à $1500 \mathrm{~m}$ d'altitude env.) et des hautes plaines. Ses exigences d'humidité et de température en font un bon indicateur climatique régional. Cependant, ses caractéristiques sont insuffisamment connues, de même que le cortège faunique dont elle fait partie au Maghreb (Bourguignat 1864; Grassé 1968).

3 Les dépôts préhistoriques renfermant des spécimens intacts de gastéropodes terrestres dans lesquels domine Leucochroa candidissima Drap. peuvent donc fournir des informations climatiques générales concernant plusieurs biotopes naturels (forestier, semi-forestier, très hygrophile, découvert, steppique sur rocher etc.). Les collections préhistoriques présentent toujours des ensembles composites, plurispécifiques, dont les besoins écologiques se chevauchent. La sélection saisonnière, fondée sur le goût, la taille, ou l'abondance, semble avoir toujours prévalu au sein d'un cortège d'espèces voisines. Dans les listes fauniques de milieux anthropiques rien ne suggère de situation d'isolat, ni de ségrégation écologique, à l'état naturel. Les associations anthropiquement créées dans les gisements expriment un savoir local, empiriquement acquis et exploité. Certes ces associations filtrent et compartimentent autrement l'environnement, ne renvoyant pas aux spécialistes de la malacofaune le cadre naturel 
complet convenant à une étude quantifiable, globale (Limondin-Lozouet 2002). Quelles informations livrent les sites préhistoriques?

\section{Comportements alimentaires}

\section{Les individus de Mechta-Afalou et les gastéropodes terrestres}

4 L'installation de la culture ibéromaurusienne* au Maghreb remonte au moins au XXII ${ }^{\mathrm{e}}$ millénaire cal BP. Portée par les Hommes de Mechta-Afalou* elle s'étend de l'Atlantique marocain à la Libye méditerranéenne et se révèle assez constante dans ses constituants anthropologiques et culturels, le choix de ses biotopes, de son genre de vie et innove magistralement dans ses comportements symboliques. Cette culture accorde aux gastéropodes terrestres et, pour la première fois à Helix aspersa Müller, une place de choix dans l'alimentation. Bien que Leucochroa candidissima Drap. ne soit pas toujours identifié, on sait, par comparaison avec l'actuel, que cette espèce lui est habituellement associée.

En Algérie littorale orientale, l'abri-nécropole d'Afalou Bou Rhummel* (Arambourg, Boule, Vallois, Verneau 1934) a livré en stratigraphie, entre 0,40 et $4,40 \mathrm{~m}$ de profondeur, divers spécimens de Leucochroa pristia Bourguignat et de Rumina decollata Linné, « les coquilles de grands Helix L. sont également abondantes » (Arambourg, p. 17 et 22). Les travaux plus récents de S. Hachi (2003, p. 48) livrent des informations concordantes: dans un foyer découvert en conditions stratigraphiques précises, plusieurs dizaines de coquilles de grands Helix L., se trouvaient réunies, certaines restées intactes (S. Hachi, en préparation).

6 L'abri voisin de Tamar-Hat, d'abord fouillé par C. Arambourg (et al. 1934), conserve la présence des mêmes espèces, parmi lesquelles domine Helix aspersa Müller (p. 29). On doit aux travaux postérieurs conduits par E. Saxon (1975), une bonne présentation quantitative de la documentation malacologique, recueillie en stratigraphie, dans les niveaux 15 à 85. Parmi d'autres espèces quantitativement moins représentées, Helix aspersa Müller domine de façon spectaculaire dans les niveaux: 7,8 (1334 et 800 individus entiers) et 15 (2604 individus).

7 Les données chronologiques (non calibrées) assignent au niveau 9 un âge de : MC 817, $16100 \pm 360$ BP., et au niveau 15, celui de : MC $812,17040 \pm 400$ BP. Or, les plus anciens spécimens recueillis dans les niveaux 84/85 (24 et 10 individus), sont datés de : MC 822, $20600 \pm 500$ BP. (Saxon 1975, p. 142).

8 Dans l'Algérois, la grotte littorale du Cap Ténès au contenu ibéromaurusien, renfermait plus de coquilles d'Helix L. que de patelles, tandis qu'à Rachgoun, en Oranie continentale, entre la Tafna et la mer, le gisement conservait plus de coquilles marines que de terrestres (Camps 1974, p. 94). Les exemples ne manquent donc pas, tous témoignent de goûts testés et comparés, révélateurs d'un éclectisme gustatif de l'homme de Mechta-Afalou, insuffisamment souligné.

9 Au Maroc oriental, l'Abri de Taforalt fouillé par l'Abbé Jean Roche a livré en contexte ibéromaurusien de nombreuses coquilles d'Helix L. (Roche 1953, 1963, et Camps 1974, p. 94). Les récents travaux conduits à Taforalt (par A. Bouzouggar), comme ceux de la grotte de Guenfouda (Oujda) le confirment (communication orale du Pr. H. Aouraghe, responsable des fouilles, mars 2005). 


\section{Les individus Proto-Méditerranéens et les gastéropodes terrestres}

10 Au début de l'Holocène, la consommation des gastéropodes terrestres s'est amplifiée avec l'entrée au Maghreb d'individus proto-Méditerranéens, porteurs de la culture capsienne. Répandue en région atlasique jusqu'au Sersou, cette culture fortement marquée, dans l'esprit des préhistoriens, par l'omniprésence des coquilles d'hélix, semblerait leur avoir accordé une place majeure. Cette observation est à l'origine de la dénomination curieuse d'«escargotières »* (Gobert 1938) qualifiant les amoncellements ainsi constitués. Quel que soit le faciès Capsien identifié (Camps 1974), chacun s'accorde à reconnaître que ce trait culturel fédérateur est largement partagé entre 8500-6000 cal BP, période de buissonnement culturel marqué par l' élassolithisme*. Les populations de faciès Columnatien* et Elassolithique*, installées en zone lagunaire sub-atlasique, ont consommé des Helix L. Mais aucun décompte n’a été publié.

$11 \mathrm{Au}$ milieu de l'Holocène, dans les gisements de Capsien supérieur, l'abondance et la diversité des espèces récoltées n'ont pas été suffisamment interprétées sur le plan climatique. Ces informations auraient pu aboutir à préciser divers biotopes, caractéristiques des régions de Sétif,Tébessa et Columnata, par exemple. Dans ces régions au climat jadis méditerranéen, l'Actuel, plus chaud et plus sec, ne renvoie qu'un paysage dégradé (Gobert et Vaufrey 1950).Toutefois, de nouvelles perspectives d'études de ce thème s'ouvrent aux recherches futures.

12 Rappelons ici l'abondante consommation par les populations du Capsien supérieur de Leucochroa candidissima Drap. et d'Helix aspersa Müller, parmi $H$. sitifensis Bourg., $H$. cespitum Drap., Rumina decollata L., Eobania constantinae Forbes (Vaufrey 1955, p. 401 ; Camps 1974, p. 168, tabl. $n^{\circ} 22$ ). En analysant avec prudence ces données quantitatives partielles, mais significatives, on soulignera l'importance des collectes dans le Constantinois et les Némencha, notamment vers Telidjène (Lubell et al 1982-1983 et Lubell 1984). Et l'on rappellera qu'aux environs de Tébessa-Gafsa, zone de forte capsianisation mi-holocène, les pionniers d'une Préhistoire régionale de référence $\mathrm{J}$. de Morgan, M. Latapie, M. Reygasse, E-G. Gobert) ne les avaient pas omises (Morel 1974, Camps et Morel 1983). Ailleurs, en Oranie, les collectes des préhistoriques ne semblent pas avoir été aussi abondantes, d'après R. de Bayle des Hermens à l'Aïn Kéda (1955, couche A : néolithique, p. 135 et B : épipaléolithique (ibéro-maurusien?, p. 159 : 52 Helix en bon état, rapportés à Rumina decollata L.et Helix melanostoma Drap.). Ces collections sont conservées à Alger au CNRPAH (ex-CRAPE).

\section{Consommation des gastéropodes par les individus Mechtoïdes et Proto-Méditerranéens durant la Néolithisation}

13 Au cours de la Néolithisation qui se répand au milieu de l'Holocène, cette pratique alimentaire survit sur les piémonts tout en s'atténuant en altitude. C'est dans le faciès du Néolithique de tradition capsienne* qu'elle se manifeste le mieux en participant au nouveau système économique qu'instaure ce faciès. L'exemple le plus précis est fourni par la grotte Capéletti* (1540 m d'alt.) du Khanguet Si Mohamed Tahar* de l'Aurès* (Roubet 2005 f). Les pasteurs de moutons, de chèvres et de bovins, venus en 
transhumance s'installer dans cette grotte du piémont nord, ont saisonnièrement occupé les alpages alentour, entre le printemps et l'automne, depuis le milieu du $\mathrm{VI}^{\mathrm{e}}$ jusqu'au III ${ }^{e}$ millénaire cal $\mathrm{BC}$. Les récoltes printanières et automnales de gastéropodes terrestres consommés en grotte semblent avoir été fréquentes, variées et assez abondantes (Roubet 1979, p. 404-409).

Fig. 1 : Grotte Capéletti : Helicidae recueillis dans les dépôts de la troisième série d'occupation
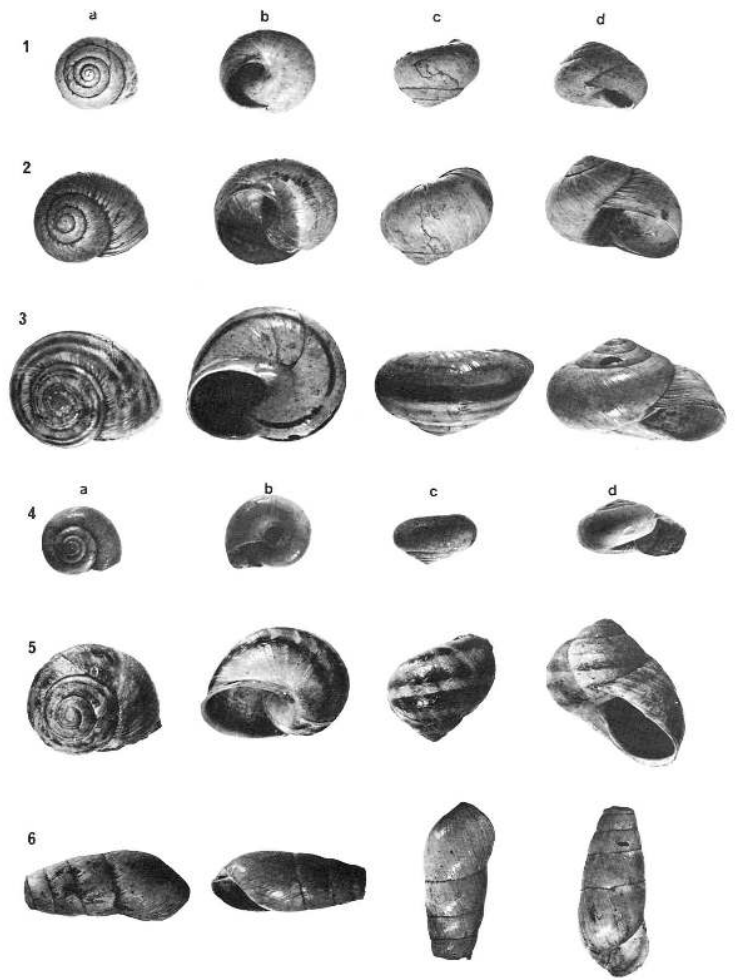

La position adoptée pour chaque espèce correspond au maintien en position de vie $(a, b)$ ou selon l'axe columellaire (c, d). Six espèces ont été identifiées ; ce sont : 1, Leucochroa candidissima ; 2, Helix melanostoma; 3 , Otala punica; 4, Helicella sitifensis ; 5 , Helix aspersa ; 6, Rumina decollata (Achatinidae). 1. Coquille lisse, luisante, blanche, ombiliquée, striée obliquement par rapport à la columelle, 5 spires : 2, coquille lisse, mate, beige, ombiliquée, stries d'accroissement orientées, irrégulières, affectées de déformations (c) visibles sur le dernier tour de spire, bouche brunâtre, 4 spires ; $\mathbf{3}$, coquille lisse, luisante, rayée de bandes plus ou moins brunes, d'épaisseur variable, stries d'accroissement orientées, très irrégulières sur le dernier tour de spire (b), 5 spires ; $\mathbf{4}$, coquille lisse, luisante, décolorée ne présentant plus que des traces de rayures irrégulières, fines, peu espacées, apex saillant, stries d'accroissement presque régulières, 5 spires ; $\mathbf{5}$, coquille bosselée, luisante, décorée, rayée de bandes brunes, variables en épaisseur, irrégulièrement espacées, stries d'accroissement irrégulières, surépaisseur localement, apex très conique, 4 spires ; $\mathbf{6}$, coquille lisse, luisante, beige, privée de sa région apicale, stries d'accroissement orientées, 4 spires visibles. (cl. J.-P. Kaufmann) (d'après Roubet 1979, p. 405)

$\mathrm{Si}$, durant la première fréquentation de la grotte cette consommation se réduit à 13 mollusques entiers, dans les séries suivantes les données quantitatives atteignent 900 spécimens dans la seconde, 6629 dans la troisième, et 604 spécimens, dans la dernière série d'occupations. Cinq espèces ont été identifiées dont Leucochroa candidissima Drap., Helix melanostoma Drap., Otala punica Morelet, Helicella sitifensis Morelet, Helix aspersa Müller et Rumina decollata L. (Fig. 1 et 2). Les fluctuations quantitatives sont difficilement explicables. Durant chaque série d'occupations, représentant 150 à 250 ans au maximum, ces récoltes peuvent aussi bien refléter des variations de goûts, que des péjorations climatiques temporaires. On remarque pourtant que Otala punica 
Morelet et Helicella sitifensis Morelet restent constamment appréciées et consommées, dans des pourcentages avoisinant les trois quarts (31,54\%) pour Otala punica Morelet, dans la troisième série. L'un de nous a tenté d'expliquer que l'alimentation des bergers - estimée d'après les déchets conservés - reposait certes sur la consommation de viande provenant de l'abattage de leurs animaux domestiques, mais s'appuyait le plus souvent, sur la diversité et l'abondance des produits de chasse et de collecte, afin de ne pas diminuer les ressources des troupeaux (Roubet 2003e, 2005f). En dehors d'un attrait gustatif certain, la valeur protéinique, nutritionnelle, de ces gastéropodes est aujourd'hui reconnue.

15 La documentation concernant la consommation des gastéropodes terrestres dans les autres faciès néolithiques du Maghreb n'a pas encore fait l'objet d'étude spécialisée.

Fig. 2 : Variation de la consommation des six espèces d'Helix durant l'occupation de la grotte Capéletti (d'après Roubet 1979, p. 407).

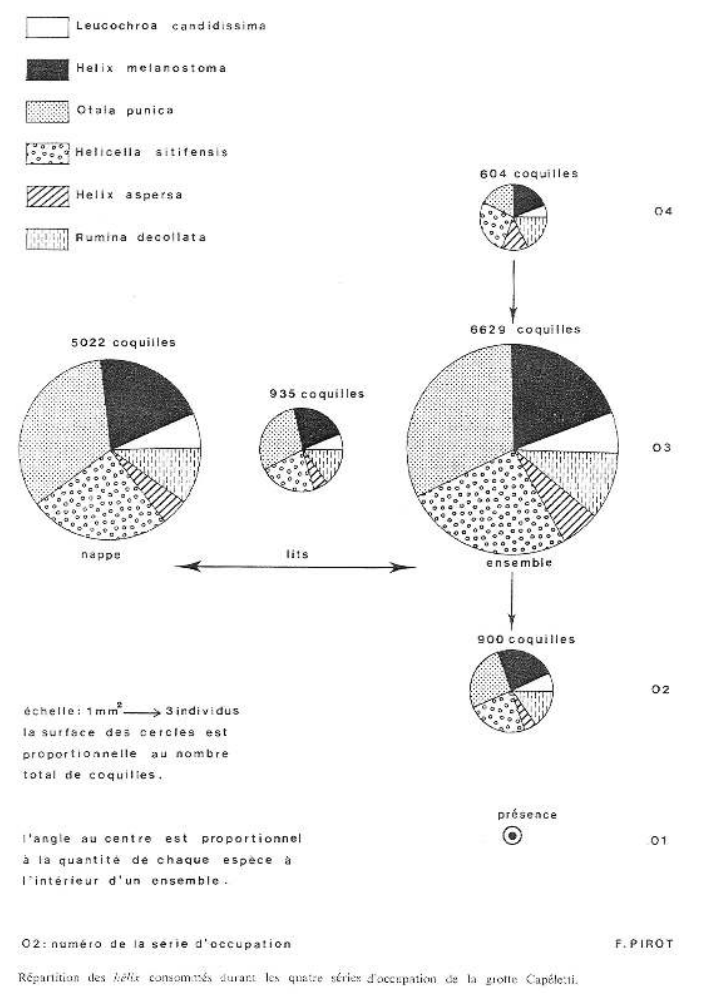

\section{Comportements symboliques}

\section{Fonction de mémoire et de témoignage de Leucochroa candidissima Drap.}

Dans le Capsien supérieur de Columnata (Tiaret), P. Cadenat $(1955,1965)$ a signalé la présence de onze coquilles d'Helix L. incisées, dont quatre perforées (Fig.3). Ces documents exceptionnels ont été délicatement entaillés jusqu'à l'opercule, sur les deux dernières spires. L'auteur a précisé qu'il s'agissait de traits parallèles de 2 à $14 \mathrm{~mm}$ de longueur, groupés, dans certains cas, par trois ou quatre. Une seule coquille présentait 25 ou 26 entailles régulières, juxta posées sur trois rangées. Les perforations sub- 
régulières étaient soignées, mais diversement localisées, l'une paraissant postérieure aux incisions. Toutes ces incisions ont été produites par le tranchant vif d'un silex, chacune segmentant presque perpendiculairement, et sans défaillance du geste $\mathrm{ni}$ repentir, les lignes de croissance de la coquille dure de l'animal. H. Camps-Fabrer (1966, p. 387) leur accorde un bref commentaire et pense qu'« on peut reconnaître dans cette disposition (des incisions (1) une inspiration comparable à celle qui a poussé les hommes capsiens à orner (2) les œufs d'autruche ».

Même si le traitement graphique de ces coquilles s'inspire techniquement de celui qu'a connu, à la même époque, la surface externe de la coquille d'œuf d'autruche, il semble, que la notion sous-jacente d'imitation ornementale, suggestive de ce rapprochement, soit acceptable mais à compléter.

Ce témoignage d'adresse technique reste unique au Maghreb, comme P. Cadenat l'a souligné, déconcertant quiconque a tenu en main de telles coquilles (Fig. 3). En effet, P. Cadenat se demande si « ces traits ne représentent pas autre chose qu'un simple motif décoratif, si, par exemple, ils n'appartiennent pas à un système figuratif dans lequel chaque ensemble aurait un sens précis, exprimerait une idée, constituerait une sorte de message facile à transmettre... un aide mémoire ou un calendrier... (Cadenat 1965, p. 317). Il semblerait que cette hypothèse soit à retenir.

Hors du domaine alimentaire, dissociées de déchets semblables rejetés, sélectionnées une à une pour que l'artiste dispose d'une série de coquilles de même gabarit, ces onze coquilles pourraient avoir été rassemblées et soigneusement modifiées, afin de porter à l'avenir un « message, mis en mémoire ». Peut-on en reconstituer le processus ?

20 Après une étape expérimentale (du geste et des incisions), pratiquée sur d'autres documents non retrouvés, il semblerait que l'artiste ait traité (nettoyé et abrasé peutêtre), une à une, la surface externe des coquilles, avant de l'utiliser comme support. Au cours de l'étape suivante l'artiste transforma chaque surface par la gravure d'incisions normées. Mais, ce que nous observons aujourd'hui représente l'état final d'une action dont le déroulement nous échappe. Quelle durée s'est-il écoulée entre la première et la dernière incision? S'agit-il du même artiste?

21 Un hiatus, n'ayant pas d'existence matérielle, s'impose à nous et vient interrompre l'idée de processus envisagée ci-dessus. En effet, ce hiatus ne permet ni d'appréhender ni de qualifier le déroulement (continu ou discontinu) de l'action de graver. Comment savoir s'il s'agit d'incisions contemporaines, gravées en une fois, ou bien d'actes échelonnés, synchronisés avec les faits devant être mémorisés? L'action perçue aujourd'hui fut précise, codée et inscrite dans une structure du message, au déroulement déterminé, tantôt continu sans hiatus, tantôt cyclique.

Comme jadis, au moment de la constitution de cette documentation, coquilles et incisions furent associées et mises en symbiose, pour former, et apparaître, aujourd'hui encore, comme un tout, indissociable. Dans cette hypothèse, la fonction de ces coquilles comme support mobilier privilégié et calibré, explicitée par l'agencement même des incisions, dut se charger d'assumer et de conserver en mémoire une sémantique singulière. 
Fig. 3 : Columnata : Capsien supérieur : coquilles d'Helix portant des incisions parallèles, groupées par trois, quatre ou cinq, à l'exception de l'une d'elles, régulièrement couverte d'incisions sur trois rangées (Fouilles et cliché P. Cadenat) ; (d'après Camps-Fabrer 1966, PI. XXXIV, p. 382).
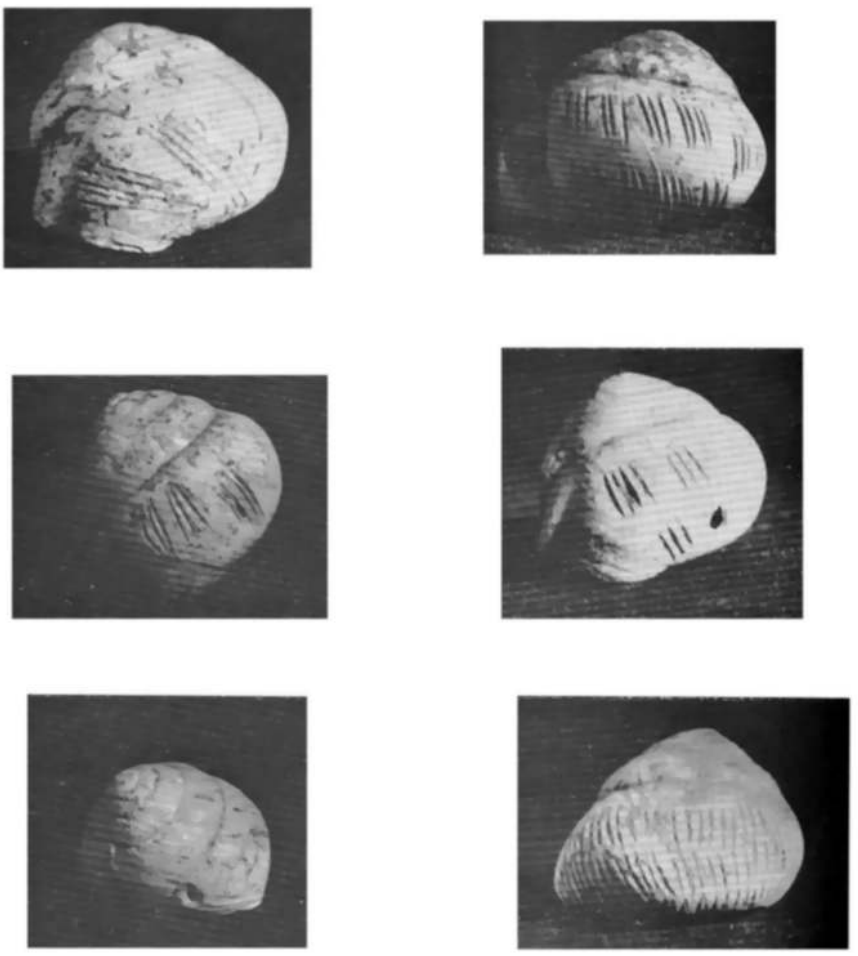

Dans l'approche des comportements symboliques, ces documents relèvent désormais d'une catégorie nouvelle celle du message, porteur d'un témoignage, en relation avec la transmission de faits, parfois décomptés. Mais a-t-on tout dit en précisant cela?

L'interrogation que soulève cette extraordinaire documentation, reste vraiment forte et tenace. En effet, ces coquilles sont désormais chargées d'un statut symbolique sublimé par ce qui paraît avoir été un " récit total ", achevé pour chacune d'elles. Voilà pourquoi chacune doit être isolément reconnue comme conservant et délivrant son message. Semblable, ou différent, nul ne peut le dire. Sur le pourtour hélicoïdal de chaque coquille, un message "conte", en décomptant. Comme si l'artiste en voulant mémoriser le dénombrement de faits semblables, savait déjà qu'il aurait à en parler en s'attardant sur chacun d'eux, pour en détailler mentalement au moins, l'aspect particulier. Dans cet esprit peut-être, dix des onze coquilles présentent des pauses, ménagées entre chaque série d'incisions. L'idée de discontinuité glissée entre des faits identiques groupés par trois, quatre ou cinq, pourrait avoir donné plus de force au vécu suggéré par l'incision et de retentissement à chaque épisode; toutes ces pauses pourraient ensuite avoir donné plus d'amplitude au message total. S'il en avait été ainsi, le message partiel que délivreraient trois, quatre, voire cinq incisions rapprochées, se composerait donc d'un écrit incisé décompté et d'espaces libres variables. Cette nouvelle forme d'écriture serait vraiment ingénieuse. D'autre part, même si le trait servant d'unité incisée, constitue à tort, pour nous, une figuration banale en apparence, il aurait scandé chaque fait de la même façon. Alors que notre regard cherche à percer aujourd'hui le mystère de chaque entaille fuselée, s'élève en nous par l'intuition d'un récit rythmé qui laisserait encore " entendre » la voix de l'artiste. Posée sur une même tonalité, la voix tantôt décompterait, puis se pauserait, tous les trois, quatre ou cinq 
temps, alors que dans un cas, la voix entamerait une longue énumération, suivant fidèlement les 25/26 incisions alignées.

Enfin, précisons que l'artiste a voulu conserver, unies et suspendues en lieu sûr (même si les notes de fouilles ne le précisent pas), quatre coquilles perforées; dans cette documentation, le trou de suspension qui ne fit pas de la coquille un objet ornemental, permit de grouper et de visualiser un ensemble cohérent, au contenu sémantique fort.

Désormais le message reste muet. La forme adoptée garde la souplesse du support hélicoïdal qui s'étire jusqu'à l'apex. Grâce à ces onze coquilles, il subsiste l'idée d'une cohérence, messagère d'observations, de rythmes cycliques, biologiques et/ou astronomiques, au schématisme sec, qui peut évoquer une comptabilité primitive. Cette émouvante documentation témoigne de comportements symboliques porteurs d'une «mémoire » transmise et recueillie aujourd'hui.

Cet essai d'interprétation souhaite rendre hommage à la perspicacité de P.Cadenat.

\section{BIBLIOGRAPHIE}

ARAMBOURG C., BOULE M., VALLOIS H., VERNEAU R., 1934. Les grottes paléolithiques des Beni Segoual (Algérie). Archives de l'I.P.H., Mémoire 13. Paris, Masson. On trouvera aussi l'étude du site de Tamar Hat, p. 25-35, 68-81.

BAYLE DES HERMENSR., 1955. « L'abri préhistorique de l'Aïn Kéda. Commune de Tiaret (département d'Oran)». Libyca, t. III, p. 129-162.

BOURGUIGNAT J.-R., 1864. Malacologie de l'Algérie ou histoire naturelle des animaux mollusques terrestres et fluviatiles recueillis jusqu'à ce jour dans nos possessions du Nord de l'Afrique. TI et II, Challamel, Paris.

CADENAT P., 1955. « Nouvelles fouilles à Columnata. Campagne de 1954-1955. Compte-rendu sommaire ». Libyca, t. III, p. 263-285.

CADENAT P., 1965. "Principaux résultats des fouilles effectuées en 1956-1957 à Columnata ». Congrés Préhistorique de France, Monaco, $\mathrm{XVI}^{\mathrm{e}}$ session, p. 316-320.

CAMPS G., 1974. Les civilisations préhistoriques de l'Afrique du Nord et du Sahara. Paris, Doin.

CAMPS G., et MOREL J., 1983. « Recherche sur l'alimentation en Afrique du Nord durant les temps épipaléolithiques ». Bulletin de la Société d'Études et de Recherches Préhistoriques, Les Eyzies, $\mathrm{n}^{\circ} 32$, p. 37-49.

CAMPS-FABRER H., 1966. Matière et art mobilier dans la Préhistoire nord-africaine et saharienne. Mémoire V du CRAPE, Paris, AMG. GOBERT E.-G., 1938. « Les escargotières. Le mot et la chose ». III Congrés de la Fédération des Sociétés Savantes de l'Afrique du Nord, Constantine, 1937, t. II, 1938, p. 639-645.

GOBERT E.-G., et VAUFREY R., 1950. « Le Capsien de l'Abri 402 ». Notes et Documents 12, Tunis, 48 p. GRASSÉ P. (sous la dir. de) 1968. Traité de Zoologie, anatomie, systématique, biologie. T. V, Mollusques gastéropodes et scaphopodes, Masson, Paris, fasc. III, p. 560 et 598-600. 
HACHI S., 2003. Les cultures de l'Homme de Mechta-Afalou. Le gisement d'Afalou-Bou Rhummel (massif des Babors,Algérie) : les niveaux supérieurs 13000-11000 BP. CNRPAH, Alger.

LIMONDIN-LOZOUET N., 2002. «Les mollusques continentaux ». in Géologie de la Préhistoire: méthodes, techniques, applications, sous la dir. de J.-C. Miskovsky, Géopré, $2^{\mathrm{e}}$ éd., p. 773-784.

LUBELL D., GAUTIER A., LEVENTAL E.T., THOMPSON M.K., SCHWARCZ H. P., SKINNER M., 1982-1983. " The prehistoric cultural ecology of Capsian escargotières : report on investigations during 1976 in the Bahiret Telidjene,Tebessa,Algeria. » Libyca, t. XXX-XXXI, p. 59-141.

LUBELL D., 1984. «Palaeoenvironments and Epi-Paleolithic economies in the Maghreb (20 000 to 5000 BP.) ». From hunters to farmers : the causes and consequences of food production in Africa. In J. Desmond Clark and Steven A. Brandt, ed. Univ. of California Press, p. 41-56.

MOREL J., 1974. « La faune de l'escargotière de Dra Mta El Ma El Abiod (Sud Algérien) : ce qu'elle nous apprend de l'alimentation et des conditions de vie des populations du Capsien supérieur ». L'Anthropologie, t. 78, p. 299-320.

ROCHE J., 1953. « La grotte de Taforalt ». L'Anthropologie, t. 57, p. 375-380.

ROCHE J., 1963. L'Epipaléolithique marocain. 2 vol. , Lisbonne.

ROUBET C., 1979. Economie pastorale en Algérie Orientale. Le Néolithique de tradition capsienne. Exemple l'Aurès. Antiquités africaines, CNRS.

ROUBET C., 2003 e. «Statut de berger » des communautés atlasiques néolithisées du Maghreb oriental, dès $7000 \mathrm{BP}$ ». L'Anthropologie, t. 107, p. 393-442.

ROUBET C.2005 f. « Khanguet Si Mohamed Tahar (Aurès) site néolithique : Aux origines du pastoralisme berbère ». EB, t. XXVII, « K52 », Édisud, p. 4205-4228.

SAXON E., 1975. Prehistoric economies of the Israeli and Algerian littorals, $18000-8000 \mathrm{BP} . \mathrm{Ph}$ D.Cambridge. On consultera en particulier p. 150-153, Tableau 2 : Faunal list :Tamar Hat Mollusca. VAUFREY R., 1955. Préhistoire de l'Afrique.T. 1, Le Maghreb. Publ. de l'Instit. des Hautes-Études de Tunis, Masson, Paris.

\section{INDEX}

Mots-clés : Alimentation, Néolithique, Préhistoire 